

\title{
BACKGROUND CONCENTRATIONS OF RADIONUCLIDES IN SOILS AND RIVER SEDIMENTS IN NORTHERN NEW MEXICO, 1974-1986
}

by

W. D. Purtymun, R. J. Peters, T. E. Buhl, M. N. Maes, and F. H. Brown

\begin{abstract}
This report documents the range and the upper limit for background concentrations of radionuclides and radioactivity in soils and river sediments that occur as natural rock-forming minerals and worldwide fallout from atmospheric nuclear weapons tests. Documentation is based on the collection of soil and sediment in northern New Mexico and analyzed for ${ }^{137} \mathrm{Cs},{ }^{238} \mathrm{Pu},{ }^{239.240} \mathrm{Pu},{ }^{90} \mathrm{Sr}$. total uranium, gross gamma, and tritium. The data used to establish the statistical range and upper limit of background concentration cover a 9- or 13-year period ending in 1986. The knowledge of background levels is necessary to interpret soil and sediment data collected for the annual environmental surveillance report and other reports relating to radionuclides or radioactivity in soils and sediments.
\end{abstract}

\section{INTRODUCTION}

Soil and river sediments are collected in and adjacent to the Los Alamos National Laboratory and analyzed for radionuclides and radioactivity as part of an ongoing environmental surveillance program. Soil and sediment samples may contain radioactivity from natural rock-forming minerals, worldwide fallout from nuclear testing or satellite burnup in the atmosphere, and in some cases, from the release of Laboratory effluents. The Laboratory's surveillance program evaluates the effects of Laboratory operations on the local and regional environment. The statistical range and the upper limit background concentration of radionuclides and radioacivity (fallout and natural) provide a baseline for comparison to interpret soil and sediment data.
Data for this compilation are for the regional and perimeter soil and sediment stations as reported in the surveillance reports for 1978 through 1986 (Environmental Surveillance Group 1979 through 1987). This study also incorporates data from a previous report by Purtymun et al. (1980) for the period 1974-1977. The regional soil and sediment stations are located in various directions from Los Alamos to provide natural and worldwide fallout background information.

Nuclear weapons testing in the atmosphere distributed ${ }^{137} \mathrm{Cs},{ }^{239,240} \mathrm{Pu},{ }^{90} \mathrm{Sr}$, and tritium worldwide as fallout. A small amount of tritium is produced by cosmic ray interaction with the atmosphere and is also distributed worldwide. It is estimated that $60 \%$ 
of ${ }^{238} \mathrm{Pu}$ is from nuclear weapons testing in the atmosphere. The remaining $40 \%$ is from burnup on reentry of sutellites containing a ${ }^{238} \mathrm{Pu}$ power source (Perkins and Thomas 1980). Some uranium in soil and sediments can be attributed to nuclear weapons testing or burnup of satellite power sources on reentry; however, most uranium in the environment is from common uranium minerals found in the earth's crust. Gross gamma radioactivity in samples from regional and perimeter stations may be related to both weapons fallout and naturally occurring radioactive minerals.

\section{A. Methods of Analysis}

Soil and sediment samples were analyzed for ${ }^{137} \mathrm{Cs},{ }^{238} \mathrm{Pu},{ }^{239.240} \mathrm{Pu},{ }^{90} \mathrm{Sr}$. total uranium, and gross gamma radioactivity. Soil moisture from soil samples was analyzed for tritium. A brief description of sample preparation and rnalyses is presented here, and a detailed description of these procedures is found elsewhere (Environmental Surveillance Group 1987).

A portion of the soil sample was removed prior to drying in the oven and soil moisture was distilled off and collected for tritium analysis. Water distilled from the soil was mixed with a counting cocktail and then analyzed for tritium by liquid scintillation counting. The remaining soils and sediments were oven dried, sieved through a $<1.7 \mathrm{~mm}$ screen, and thoroughiy blended. Separate aliquots of the prepared sample were taken for each analysis.

Samples for ${ }^{137} \mathrm{Cs}$ were counted directly with a $\mathrm{Ge}(\mathrm{Li})$ detector coupled with a multichannel analyzer. Aliquots (10 g) for ${ }^{238} \mathrm{Pu}$ and ${ }^{239,240} \mathrm{Pu}$ analysis were spiked with ${ }^{242} \mathrm{Pu}$ tracers and dissolved in acid. Plutonium was isolated by anion exchange and electroplated onto a stainless steel disk. The disk was then counted on an alpha spectrometer.
Samples for total uranium from 1974 through 1976 were performed using a fluorometric method. Total uranium analyzed from 1976 through 1986 was performed by irradiating the sample with epithermal neutrons, followed by counting on a $\mathrm{Ge}(\mathrm{Li})$ gamma ray spectrometer. This change in analytical method for total uranium resulted in a change in the limit of detection (Table 1).

Samples for ${ }^{90} \mathrm{Sr}$ analysis were dissolved in acid, then extracted with bis(2-ethyl hexyl) hydrogen phosphate to remove ${ }^{90} Y$ daughters and interfering radionuclides. Stable yttrium was added as a chemical tracer. After allowing the $90 y$ daughter to equilibrate with ${ }^{90} \mathrm{Sr}$, the sample was extracted, the yttrium purified, and the sample counted on a gas-proportional counter.

Gross gamma analysis is instrument specific: thus the measurements reported are only relative to that instrument. Gross gamma data were used for scieening soil and sediment samples. The data may not be of interest relative to data generated by another laboratory; however, it is important to establish the range and upper background for gross gamma data generated here to interpret the results of the screening process. Samples for gross gamma analy ses were counted directly in a Nal(TI) we!l counter

\section{B. Interpretation of Data}

There have been no significant long-term trends or changes noted in concentrations for specific radionuclides for the 9- or 13-year period ending in 1986. However, there appear to be some statistical differences in concentrations for certain radionuclides and radioactivity among some but not all stations. The differences may be due to regional and

Table I. Limits of Detection

${ }^{137} \mathrm{Cs}$
${ }^{238} \mathrm{Pu}$
${ }^{239} \cdot 2+0 \mathrm{Pu}$
${ }^{90} \mathrm{Sr}$
Total uranium (1974-76)
Total uranium (1976-86)
Gross gamma
Tritium

$$
\begin{aligned}
& 0.10 \mathrm{pCi} / \mathrm{g} \\
& 0.002 \mathrm{pCi} / \mathrm{g} \\
& 0.002 \mathrm{pCi} / \mathrm{g} \\
& 0.05 \mathrm{pCi} / \mathrm{g} \\
& 1, \mathrm{~g} / \mathrm{g} \\
& 0.1 \mu \mathrm{g} / \mathrm{g} \\
& 0.1 \mathrm{counts} / \mathrm{min} / \mathrm{g} \\
& 0.3 \times 10^{-6} \mu \mathrm{Ci} / \mathrm{ml}
\end{aligned}
$$


local weather patterns and elevation of stations re. lated to worldwide fallout. They may also be at tributed to changes in concentration of natural radioactivity in local soils $\mathrm{c}^{r}$ sediments. Results showing statistical similarities or differences of analysis from various stations are summarized in Appendix A.

The limits of detection for various radionuclides and radioactivity are affected by sample preparation. the mass of sample, couriting time, and counter etficiency and background. As a number of different counters are used for analysis of the same radionuclide. slightly different results can be obtained for the same sampie counted in different counters. The background analytical results are reported in very low concentrations, near the limits of detection. Thus, the analytical results from the same station may or may not have a normal distribution from the variation in counter efficiency

Analytical results for which counter or instrument background must be subtracted may result in a net value that is lower than the limits of detection and may result in values of zero or negative numbers The negative numbers are not a physical reality; however, a valid long-term mean can be obtained only if very small negative and positive values are included in a sample set to obtain a statistical range and upper limit background concentrations.

Analytical data for the 9- or 13-year period ending in 1986 were used to determine the statistical range and upper limit background concentrations for ${ }^{137} \mathrm{Cs}$. ${ }^{238} \mathrm{Pu} .{ }^{239.240} \mathrm{Pu}$, total uranium, gross gamma, and tritium. The soil and sediment data for these periods provide the longest record for collection and analysis from the same stations using comparable sampling and analytical techniques. The mean plus or minus twice the standard deviation $(\bar{X} \pm 2 s)$ de rived from the period of record for the various radionuclides and radioactivity was used to establish a statistical range for normal background concentrations. The mean plus twice the standard deviation $(\bar{X}+2 s)$ is the upper limit background concentration for that particular radionuclide. The upper limit background level calculated this way leads to the statistical expectation that only 1 in 40 background samples analyzed will exceed the upper background concentration. The data are summarized in Table II by showing radionuclides, number of analyses, mini- mum, maximum, mean $(\bar{X})$, standard deviation (s), and the upper limit background concentration ( $\bar{X}$. 2s)

\section{SOILS}

Soil stations are located near Chamita, Embudo, Cochiti, Bernalillo, and Jemez (Fig. 1). A soil ring 7.5 $\mathrm{cm}$ in diameter driven $5.0 \mathrm{~cm}$ into the soil was used to collect the sample. Samples were collected from the center and corners of a square area $10 \mathrm{~m}$ per side. The five samples were combined to form a composite sample for analysis. Soil data are shown by station, month and year, and by radionuclides or radioactivity in Appendix $B$.

There were 64 soil samples taken from 5 stations analyzed for ${ }^{137} \mathrm{Cs}$ during the period 1974-1986 The concentrations ranged from 0.00 to $1.4 \mathrm{pCi} / \mathrm{g}$ with an upper limit background concentration of 1.09 $\mathrm{pCi} / \mathrm{g}$ (Table II).

There were 76 soil samples from 5 stations analyzed for plutonium. The ${ }^{238} \mathrm{Pu}$ ranged from -0.005 to $0.010 \mathrm{pCi} / \mathrm{g}$ with an upper limit background of $0.005 \mathrm{pCi} / \mathrm{g}$. The ${ }^{239,240} \mathrm{Pu}$ concentrations were slightly greater, ranging from -0.002 to $0.052 \mathrm{pCi} / \mathrm{g}$ with an upper limit background of $0.025 \mathrm{pCi} / \mathrm{g}$.

There were 29 soil samples from 5 stations ana lyzed for ${ }^{90} \mathrm{Sr}$ with an upper limit background concentration of $0.88 \mathrm{pCi} / \mathrm{g}$; 34 soil samples were analyzed for total uranium with an upper limit background of $3.4 \mu \mathrm{g} / \mathrm{g}$ : 39 soil samples were analyzed for gross gamma radioactivity with an upper limit background of 6.6 counts $/ \mathrm{min} / \mathrm{g}$; and 43 soil nois. ture samples were analyzed for tritium $\left({ }^{3} \mathrm{H}\right)$ with an upper limit background concentration of $7.2 \times 10^{-5}$ ${ }{ } \mathrm{Ci} / \mathrm{ml}$.

\section{PIVER SEDIMENTS}

Radionuclides have an affinity for silts and clays in the soils. These soils and attached radionuclides are subject to sheet erosion and transport into streams and rivers. The main channels of rivers in the Los Alamos area generally consist of cobbles and boulders, whereas the finer sediments occur as dune build-up behind large boulders in the channel or along the edges of the channel. Sediments from 
Iable 11. Summary of Worldwide Fallout and/or Natural Levels of Radionuclides in the Enviranment

\begin{tabular}{|c|c|c|c|c|c|c|c|}
\hline & Units & $\begin{array}{c}\text { Number } \\
\text { of } \\
\text { Analyses }\end{array}$ & Minimulli & Maxinfum & $\begin{array}{r}\text { Mean } \\
(\bar{x}) \\
\end{array}$ & $\begin{array}{l}\text { Standard } \\
\text { Deviation } \\
\text { (s) }\end{array}$ & $\begin{array}{l}\text { Upper Limit } \\
\text { Background } \\
(\bar{x}+25)\end{array}$ \\
\hline \multicolumn{8}{|l|}{ Soils } \\
\hline \multirow{4}{*}{$\begin{array}{l}137{ } \mathrm{Cs} \\
238 \mathrm{Pu} \\
239,240_{\mathrm{Pu}} \\
90_{\mathrm{Sr}}\end{array}$} & $\mathrm{pCi} / \mathrm{g}$ & 64 & $0.00(0.10)$ & $1.4(0.08)$ & 0.43 & 0.33 & 1.09 \\
\hline & $p C i / g$ & 76 & $.0 .005(0.002)$ & $0.010(0.006)$ & 0.001 & 0.002 & 0.005 \\
\hline & $p C i / g$ & 76 & $.0 .002(0.010)$ & $0.052(0.006)$ & 0.007 & 0.009 & 0.025 \\
\hline & $\mathrm{pCi} / \mathrm{g}$ & 29 & $0.03(0.03)$ & $1.0(1.2)$ & 0.34 & 0.27 & 0.88 \\
\hline Total uranium & $1+9 / 9$ & 34 & $1.3(0.2)$ & $3.9(0.2)$ & 2.4 & 0.5 & 3.4 \\
\hline \multirow{2}{*}{$\begin{array}{l}\text { Gross gamma } \\
3_{H}\end{array}$} & Counts/min/g & 39 & $-0.2(0.06)$ & $8.5(0.30)$ & 3.2 & 1.7 & 0.6 \\
\hline & $10^{-6}, \mathrm{Ci} / \mathrm{ml}$ & 43 & $-0.8(0.3)$ & $8.8(0.9)$ & 2.6 & 2.3 & 7.2 \\
\hline \multicolumn{8}{|l|}{ Sediments } \\
\hline${ }_{238}^{137} \mathrm{Cs}$ & $\mathrm{pCi} / \mathrm{g}$ & 103 & $-0.01(0.06)$ & $0.82(0.08)$ & 0.18 & 0.13 & 0.44 \\
\hline${ }_{238}^{234} \mathrm{Pu}$ & $\mathrm{pCi} / \mathrm{g}$ & 113 & $.0 .020(0.030)$ & $0.012(0.010)$ & 0.000 & 0.003 & 0.006 \\
\hline $239,240 \mathrm{Pu}$ & $\mathrm{pCi} / \mathrm{g}$ & 113 & $.0 .013(0.008)$ & $0.065(0.005)$ & 0.005 & 0.009 & 0.023 \\
\hline${ }^{2} \mathrm{Sr}$ & $\mathrm{pCi} / \mathrm{g}$ & 36 & $.0 .16(0.10)$ & $1.1(0.06)$ & 0.23 & 0.32 & 0.87 \\
\hline Total uranium & $/ 9 / 9$ & 59 & $0.7(0.1)$ & $5.8(0.8)$ & 2.6 & 0.9 & 4.4 \\
\hline Grosis gimmä & Counts/min/g & 62 & $-4.8(0.80)$ & $8.6(0.15)$ & 2.9 & 2.5 & 7.9 \\
\hline
\end{tabular}

Note: Analytical uncertaintios in parentheses. 


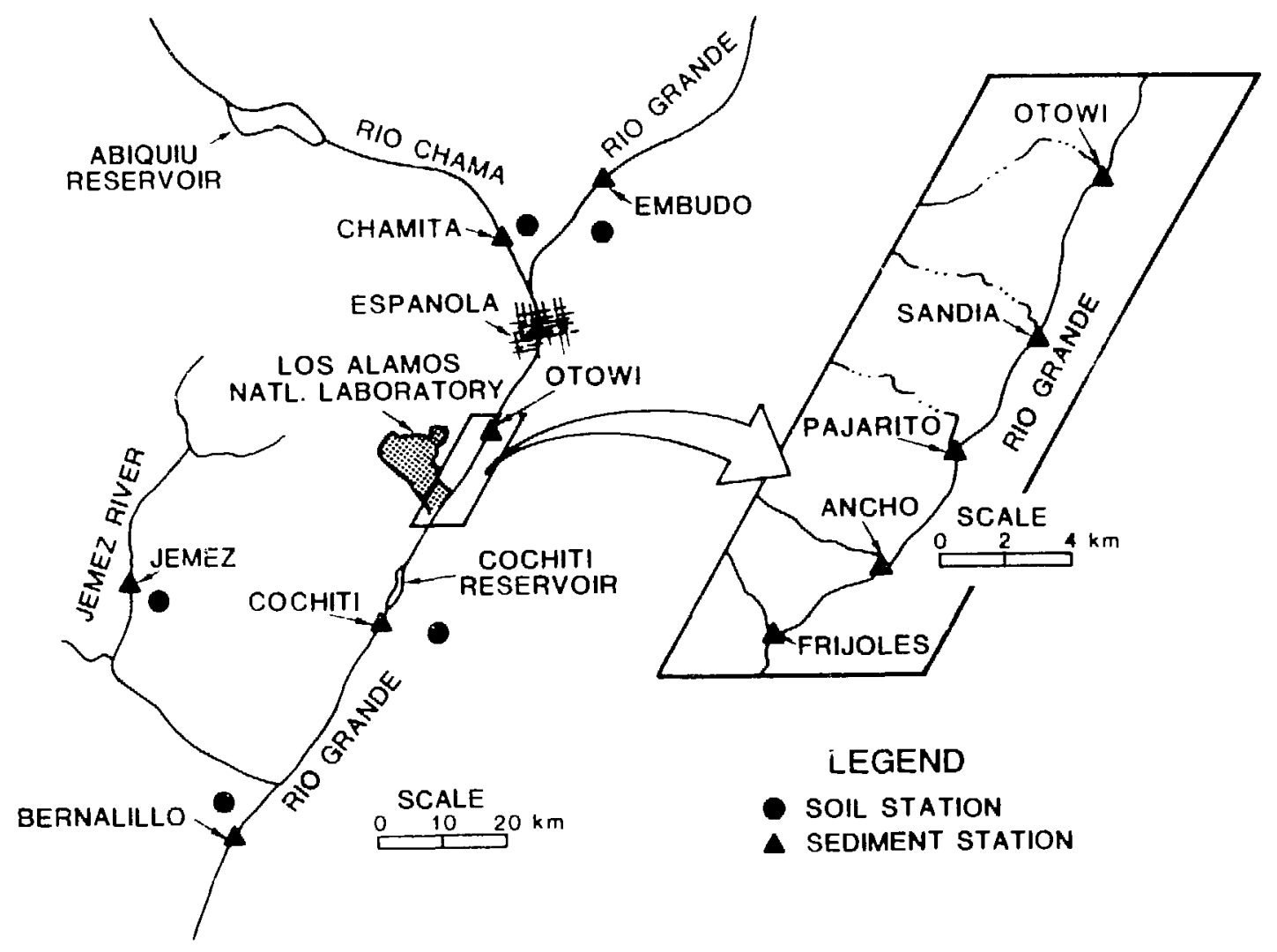

Figure 1. Location of Soil and Sediment Stations.

dunes or along tha edge of the channel are collected for analyses as the fine sediments are most representative of the sediment transport of radionuclides.

The river sediment stations are located on the Rio Chama near Chamita, on the Rio Grande at Embudo and Otowi, on the Rio Grande below Sandia, Pajarito, Ancho, and Frijoles Canyons, on the Rio Grande at Bernalillo, and on the Jemez River riear Jemez (Fig. 1). Sediment data are shown in Appendix C.

There were 103 sediment samples from 9 stations analyzed for ${ }^{137} \mathrm{Cs}$. The concentrations ranged from -0.01 to $0.82 \mathrm{pCi} / \mathrm{g}$ with an upper limit background concentration of $0.44 \mathrm{pCi} / \mathrm{g}$ (Table II).

There were 113 sediment samples from 9 stations analyzed for plutonium. The ${ }^{238} \mathrm{Pu}$ concentrations ranged from -0.020 to $0.012 \mathrm{pCi} / \mathrm{g}$ with an upper limit background concentration of $0.006 \mathrm{pCi} / \mathrm{g}$. The ${ }^{239,240} \mathrm{Pu}$ concentrations were slightly greater, ranging from -0.013 to $0.065 \mathrm{pCi} / 9$ with an upper limit background of $0.023 \mathrm{pCi} / \mathrm{g}$.

There were 36 sediment samples analyzed for ${ }^{90} \mathrm{Sr}$ with an upper limit background concentration of $0.87 \mathrm{pCi} / \mathrm{g} ; 59$ analyses for total uranium, with an upper limit background of $4.4 \mathrm{\mu g} / \mathrm{g}$ and 62 analyses for gross gamma radioactivity with an upper limit background concentration of 7.9 counts $/ \mathrm{min} / \mathrm{g}$.

\section{REFERENCES}

Environmental Surveillance Group, "Environmental Surveillance at Los Alamos During 1978," Los Alamos Scientific Laboratory report LA-7800-ENV (1979).

Environmental Surveillance Group, "Environmental Surveillance at Los Alamos During 1979," Los Alamos Scientific Laboratory report LA-B200-ENV (1980). 
Environmental Surveillance Group, "Environmental Surveillance at Los Alamos During 1980," Los Alamos National Laboratory report LA-8810-ENV (1981).

En،,ıronmental Surveillance Group, "Environmental Surveillance at Los Alarnos During 1981," Los Alamos National Laboratory report LA-9349-ENV (1982).

Environmental Surveillance Group, "Environmental Surveillance at Los Alamos During 1982," Los Alamos National Laboratory report L4-9762-ENV (1983).

Environmental Surveillance Group. "Environmental Surveillance at Los Alamos During 1983," Los Alamos National Laboratory report LA-10100-ENV (1984).

Environmental Surveillance Group, "Environmental Surveillance at Los Alamos During 1984," Los Alamos National Laboratory report LA-10421-ENV (1985).
Environmental Surveillance Group, "Environmental Surveillance at Los Alamos During 1985," Los Alamos National Laboratory report LA-10721-ENV (1986).

Environmental Surveillance Group, "Environmental Surveillance at Los Alamos During 1986, "Los Alamos National Laboratory report LA-10992-ENV (1987).

R. W. Perkins and C. W. Thomas, "Worldwide Fallout," in Transuranic Elements in the Environment, Technical Information Center, U.S. Dept. of Energy (1980).

W. D. Purtymun, R. J. Peters, and A. K. Stoker, "Radioactivity in Soils and Sediments in and Adjacent to the Los Alamos Area, 1974-77," Los Alamos Scientific Laboratory report LA-8234-MS (1980). 


\section{APPENDIX A}

To determine the influence of the station focation on radionuclide concentrations, both a parametric test (One-Way Analysis of Varıance) for normal populations and a nonparametric test (Kruskal-Wailis) for nonnormal populations were used on soil and sediment data. The results of the tests for soil and sediments are summarized in Table A-I. As described in Section I.B, "Interpretation of Data," differences in concentrations of radionuclides from station to station may be related to regional and local weather patterns, elevation of the stations related to worldwide fallout, or changes in concentration of natural radioactivity in local minerals found in soils and sediments.

In both cases the hypothesis to be tested was that there was no significant difference for analytical results between different stations for various sample types. When there were significant differences between stations, the bias was considered "positive." When there were no significant differences between stations, the bias was considered to be "negative." In one case (uranium in sediments) the results were indeterminate, as the test statistics were borderline.

There was strong positive station bias (significant difference in concentration between stations) for ${ }^{137} \mathrm{Cs}$. ${ }^{239,240} \mathrm{Pu}$, total uranium, and gross gamma in soils, and ${ }^{137} \mathrm{Cs}$ and gross gamma in sediments. There was a negative station bias (no significant difference in concentrations between stations) for ${ }^{238} \mathrm{Pu}$ and ${ }^{90} \mathrm{Sr}$ in soils and ${ }^{238} \mathrm{Pu},{ }^{239.240} \mathrm{Pu}$, and ${ }^{90} \mathrm{Sr}$ in sediments.

Cesium and gross gamma in both sediments and soils indicate a strong positive station bias suggesting that they undergo limited transport afte: deposition. Their distribution probably reflects the effects of local conditions.

Uranium concentrations in soil indicated a strong positive station bias that would be expected as they reflect differences in natural uranium concentration, in parent rock material, or in soil. The tests for uranium in sediments were indeterminate, indicating a neutral area between a positive and negative bias between sediment stations or no correlation of concentrations between stations.

The background plutonium found in the regional and perimeter stations is the result of worldwide failout. The worldwide fallout concentrations of plutorium in soils may vary with regional and local wind patterns and differences in elevations of the stations. The ${ }^{239,240} \mathrm{Pu}$ in soils reflects this difference with a positive station bias (difference between stations).

The ${ }^{238} \mathrm{Pu}$ in soils or sediments does not reflect this difference as would be expected The tests indicate a negative station bias (no differences between stations). Background or fallout concentrations of ${ }^{238} \mathrm{Pu}$ are typically much less than ${ }^{239.240} \mathrm{Pu}$ in soils or sediments with an average ${ }^{239,240} \mathrm{Pu} /{ }^{238} \mathrm{Pu}$ ratio in the area of about 20 (Environmental Surveillance Group 1985). This value was derived from large samples ( $\mathrm{kg}$ ) collected and analyzed that resulted in a lower limit of detection than data presented in this report. The ratio for data in this report is about 5 (upper background). This is primarily because most of the ${ }^{238} \mathrm{Pu}$ values presented in this report are at or below the analytical limits of detection, which do not represent a real number but a statistical number. The fact that the plutonium ratios in this report are low and the tests indicated negative station bias (no difference in concentrations between station) is probably attributed to th: ${ }^{238} \mathrm{Pu}$ analytical limits of detection masking any real variation in the actual concentrations.

The concentrations of ${ }^{239,240} \mathrm{Pu}$ and ${ }^{90} \mathrm{Sr}$ in sediments indicate a negative station bias (no significant difference in concentrations between stations). This is as exp acted with sediment samples, where transport and mixing within the streams effectively mask any significant difference between sample station.

The ${ }^{90} \mathrm{Sr}$ concentrations in both soils and sediments show strong negative station bias indicating a uniform deposition in soil and transport as sediments. 
Table A-I. Statistical Analyses of Results of Radioactivity from Various Stations

\begin{tabular}{|c|c|c|c|c|c|}
\hline \multirow[b]{2}{*}{ Nuclide } & \multicolumn{2}{|c|}{ Anova } & \multicolumn{2}{|c|}{ Kruskal-Wallis } & \multirow{2}{*}{$\frac{\text { Site Station }}{(x=0.05)}$} \\
\hline & d.f. $^{a}$ & F-ratio & d.f. $^{a}$ & $\mathrm{KW}$ & \\
\hline & \multicolumn{5}{|c|}{ Soil Samples } \\
\hline${ }^{137} \mathrm{Cs}$ & $4 / 59$ & 20.69 & 4 & 35.89 & POS \\
\hline${ }^{238} \mathrm{Pu}$ & $4 / 71$ & 1.13 & 4 & 5.30 & NEG \\
\hline${ }^{238,240} \mathrm{Pu}$ & $4 / 71$ & 7.15 & 4 & 31.35 & POS \\
\hline${ }^{90} \mathrm{Sr}$ & $4 / 24$ & 0.50 & 4 & 3.36 & NEG \\
\hline Uranium & $4 / 29$ & 5.10 & 4 & 16.02 & POS \\
\hline Gamma & $4 / 34$ & 5.07 & 4 & 18.71 & POS \\
\hline \multirow[t]{2}{*}{ Tritium } & $4 / 38$ & 0.47 & 4 & 1.91 & NEG \\
\hline & & \multicolumn{4}{|c|}{ Sediment Samples } \\
\hline${ }^{137} \mathrm{Cs}$ & $8 / 86$ & 2.93 & 8 & 19.73 & POS \\
\hline${ }^{238} \mathrm{Pu}$ & $8 / 96$ & 1.03 & 8 & 3.82 & NEG \\
\hline${ }^{238,240} \mathrm{Pu}$ & $8 / 95$ & 1.72 & 8 & 11.36 & NEG \\
\hline${ }^{90} \mathrm{Sr}$ & $8 / 26$ & 0.58 & 8 & 8.83 & NEG \\
\hline Uranium & $8 / 44$ & 1.85 & 8 & 16.95 & IND \\
\hline Gamma & $7 / 48$ & 2.34 & 8 & 20.70 & POS \\
\hline
\end{tabular}


Appendix $B$

Radiochemical Analyses of Soil Showing fallout and/or Natural Levels

\begin{tabular}{|c|c|c|c|c|c|c|c|c|}
\hline $\begin{array}{c}\text { Soil Stations } \\
\text { (near) }\end{array}$ & $\begin{array}{l}\text { Manth } \\
\text { Year } \\
\end{array}$ & $\begin{array}{c}137 \mathrm{cs} \\
(\mathrm{pCi} / \mathrm{g})\end{array}$ & $\begin{array}{l}{ }_{23 B_{\mathrm{Pu}}} \\
(\mathrm{pCi} / \mathrm{g})\end{array}$ & $\begin{array}{c}239,240 \mathrm{Pu} \\
(\mathrm{pC} \mathrm{i} / \mathrm{g}) \\
\end{array}$ & $\begin{array}{r}90 \mathrm{sr} \\
(\mathrm{pCig} /) \\
\end{array}$ & $\begin{array}{c}\text { Total Uranium } \\
(419 / 9) \\
\end{array}$ & $\begin{array}{c}\text { Gross Gamma } \\
\text { (Counts/min/g) }\end{array}$ & $\begin{array}{c}3_{H} \\
(10.6 \mu[\mathrm{i} / \mathrm{ml})\end{array}$ \\
\hline \multirow[t]{16}{*}{ Chamita } & $7 \cdot 74$ & $0.81(0.13)$ & $0.001(0.001)$ & $0.006(0.002)$ & $\cdots$ & $\cdots$ & $\cdots$ & $\cdots$ \\
\hline & $5 \cdot 75$ & .. & - & . & $\because$ & $\cdots$ & $\cdots$ & . \\
\hline & 10.57 & .. & $0.000(0.001)$ & $0.017(0.003)$ & $\cdots$ & .. & $\cdots$ & $\cdots$ \\
\hline & $3 \cdot 76$ & $0.68(0.03)$ & $0.001(0.001)$ & $0.008(0.002)$ & . & .. & -. & $\cdots$ \\
\hline & $10 \cdot 76$ & .. & $0.002(0.002)$ & $0.014(0.002)$ & $0.38(0.16)$ & $\cdots$ & $\cdots$ & $\cdots$ \\
\hline & $3-77$ & $0.75(0.06)$ & $\cdot 0.002(0.001)$ & $0.017(0.003)$ & $0.09(0.07)$ & $\cdots$ & $\cdots$ & $\cdots$ \\
\hline & $10 \cdot 77$ & $0.57(0.08)$ & $-0.001(0.000)$ & $0.010(0.003)$ & $0.29(0.09)$ &.. & . & . \\
\hline & $3-78$ & $0.68(0.06)$ & $0.000(0.001)$ & $0.013(0.002)$ & .. & . & $3.7(0.12)$ & $5.8(0.4)$ \\
\hline & $3-79$ & $0.82(0.07)$ & - & - & $0.44(0.08)$ & $2.3(0.2)$ & . & $1.3(0.3)$ \\
\hline & $2 \cdot 80$ & $0.67(0.06)$ & $0.000(0.002)$ & $0.005(0.002)$ & $0.43(0.10)$ & $2.3(0.2)$ & $3.5(0.12)$ & $2.2(0.3)$ \\
\hline & $3 \cdot 81$ & $0.69(0.06)$ & $0.010(0.006)$ & $0.013(0.003)$ & $0.45(0.10)$ & . & $3.8(0.10)$ & $0.2(0.3)$ \\
\hline & $3 \cdot 82$ & $0.73(0.04)$ & $0.001(0.001)$ & $0.019(0.003)$ & - & $3.2(0.3)$ & . & $2.2(0.3)$ \\
\hline & $2 \cdot 83$ & $0.14(0.03)$ & $0.003(0.000)$ & $0.000(0.000)$ & $\therefore$ & $3.2(0.3)$ & $1.6(0.10)$ & $1.4(0.3)$ \\
\hline & $2 \cdot 84$ & $0.80(0.05)$ & $0.001(0.001)$ & $0.020(0.003)$ & .. & $3.9(0.2)$ & $3.7(0.30)$ & $1.5(0.3)$ \\
\hline & $3 \cdot 85$ & $0.67(0.07)$ & $0.000(0.001)$ & $0.014(0.003)$ & $\cdots$ & $3.1(0.2)$ & $3.4(0.40)$ & $.0 .8(0.3)$ \\
\hline & $2-86$ & $0.25(0.04)$ & $0.001(0.001)$ & $0.003(0.001)$ & -. & $1.7(0.2)$ & $1.2(0.30)$ & $6.4(0.8)$ \\
\hline \multirow[t]{16}{*}{ Embudo } & $7 \cdot 74$ & $0.56(0.13)$ & $0.001(0.001)$ & $0.005(0.001)$ & $\cdots$ & -. & . & $\cdots$ \\
\hline & $5 \cdot 75$ & . & $0.000(0.001)$ & $0.008(0.002)$ & $\because$ & $\cdots$ & $\cdots$ & $\cdots$ \\
\hline & $11 \cdot 75$ & . & $0.000(0.001)$ & $0.009(0.002)$ & $\cdots$ & -. & . & .. \\
\hline & $3 \cdot 76$ & $0.77(0.03)$ & $0.001(0.001)$ & $0.012(0.002)$ & $0.90(1.0)$ &.. & - & . \\
\hline & $10 \cdot 76$ & . & $0.003(0.002)$ & $0.019(0.003)$ & - & .. & .. & .. \\
\hline & 3.77 & $0.37(0.03)$ & $0.001(0.005)$ & $0.010(0.005)$ & $0.40(0.1)$ & . & . & $\cdots$ \\
\hline & $10 \cdot 77$ & $0.72(0.08)$ & $0.002(0.002)$ & $0.015(0.006)$ & . & . & - & $\cdots$ \\
\hline & $3 \cdot 78$ & $1.2(0.20)$ & .. & . & . & . & $3.4(0.11)$ & . \\
\hline & $3-79$ & $0.88(0.09)$ & $0.000(0.002)$ & $0.020(0.003)$ & $0.26(0.07)$ & $3.3(0.3)$ & $1.4(0.12)$ & $0.6(0.3)$ \\
\hline & $2 \cdot 80$ & $0.79(0.09)$ & $0.001(0.002)$ & $0.017(0.005)$ & $0.33(0.08)$ & $2.1(0.2)$ & $3.0(0.11)$ & $2.5(0.3)$ \\
\hline & $3 \cdot 81$ & $1.1(0.11)$ & $0.001(0.001)$ & $0.021(0.004)$ & $0.30(0.06)$ & . & $3.7(0.12)$ & $1.2(0.3)$ \\
\hline & $3 \cdot 82$ & $1.4(0.08)$ & $0.004(0.001)$ & $0.029(0.004)$ & . & $2.2(0.2)$ & . & $3.7(0.3)$ \\
\hline & 2.83 & $0.04(0.04)$ & $0.006(0.003)$ & $0.001(0.001)$ & & $2.6(0.3)$ & $7.9(0.14)$ & $1.4(0.3)$ \\
\hline & $2 \cdot 84$ & $0.90(0.15)$ & $0.000(0.001)$ & $0.016(0.003)$ & . & $2.2(0.1)$ & $8.5(0.30)$ & $5.4(0.6)$ \\
\hline & $3 \cdot 85$ & $0.94(0.18)$ & $0.001(0.001)$ & $0.018(0.003)$ & $\cdots$ & $2.2(0.2)$ & $3.2(0.40)$ & $.0 .7(0.3)$ \\
\hline & $2 \cdot 86$ & $0.69(0.13)$ & $0.001(0.002)$ & $0.017(0.003)$ & .. & $2.4(0.2)$ & $3.7(0.50)$ & $0.8(0.4)$ \\
\hline
\end{tabular}




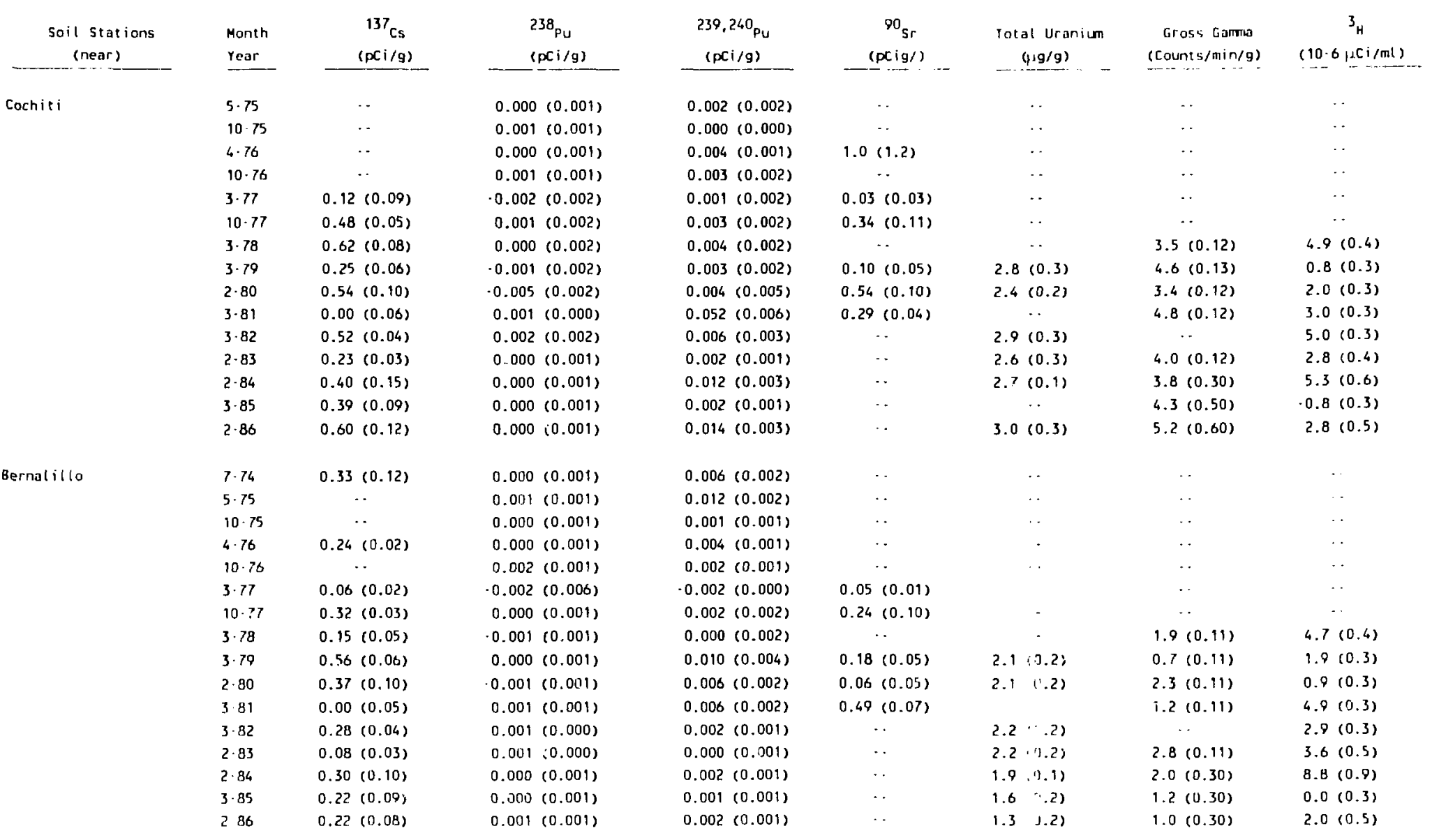


Appendt 8 (cont)

\begin{tabular}{|c|c|c|c|c|c|c|c|c|}
\hline $\begin{array}{c}\text { Soll stations } \\
\text { (near) }\end{array}$ & $\begin{array}{l}\text { Month } \\
\text { Year }\end{array}$ & $\begin{array}{c}{ }^{137} \mathrm{Cs} \\
(p<i / g)\end{array}$ & $\begin{array}{l}238 \mathrm{Pu} \\
(\mathrm{pC} / / \mathrm{g})\end{array}$ & $\begin{array}{c}239.240 \mathrm{Pu} \\
(\mathrm{p} C \mathrm{i} / \mathrm{g})\end{array}$ & $\begin{array}{c}90_{s r} \\
(p<i g /)\end{array}$ & $\begin{array}{c}\text { Total Uranium } \\
(1 / 9 / 9)\end{array}$ & $\begin{array}{c}\text { Gross Gamma } \\
\text { (counts/min/g) }\end{array}$ & $\begin{array}{c}{ }^{3} \mathrm{H} \\
(10-6 \mu \mathrm{Ci} / \mathrm{ml}) \\
\end{array}$ \\
\hline \multirow[t]{16}{*}{ Jemet } & 7.74 & $0.11(0.12)$ & $0.000(0.000)$ & $0.001(0.002)$ & .. & $\cdots$ & $\cdots$ & .. \\
\hline & $5 \cdot 75$ & . & $0.002(0.001)$ & $0.001(0.001)$ & .. & $\cdots$ & $\cdots$ & .. \\
\hline & 9.75 & .. & $.0 .001(0.001)$ & $0.012(0.003)$ & . & & .. & . \\
\hline & $4-78$ & $0.11(0.02)$ & $0.001(0.001)$ & $0.002(0.001)$ & $0.98(1.2)$ & $\cdots$ & $-\cdot$ & .. \\
\hline & $10 \cdot 76$ & $0.14(0.02)$ & $0.001(0.001)$ & $0.007(0.002)$ & $0.05(0.20)$ & $\cdots$ & $\cdots$ & $\cdots$ \\
\hline & $3 \cdot 77$ & $0.21(0.05)$ & $0.010(0.030)$ & $-0.020(0.030)$ & $0.14(0.04)$ & . & $\therefore$ & . \\
\hline & $10 \cdot 77$ & $0.07(0.06)$ & $0.000(0.002)$ & $0.001(0.002)$ & $0.26(0.11)$ & .. & .. & $\cdots$ \\
\hline & $3 \cdot 78$ & $0.06(0.14)$ & $.0 .002(0.001)$ & $0.001(0.001)$ & . & . & $2.7(0.11)$ & . \\
\hline & $3 \cdot 79$ & $0.33(0.07)$ & $-0.001(0.001)$ & $0.005(0.002)$ & $0.17(0.07)$ & $2.0(0.2)$ & $.0 .2(0.06)$ & $0.0(0.3)$ \\
\hline & $2 \cdot 80$ & $0.18(0.07)$ & $.0 .002(0.001)$ & $0.003(0.003)$ & $0.63(0.13)$ & $2.4(0.2)$ & $37(0.12)$ & $0.8(0.3)$ \\
\hline & $3 \cdot 81$ & $0.23(0.05)$ & $0.001(0.001)$ & $0.002(0.001)$ & $0.07(0.19)$ & . & $2.5(0.11)$ & $5.8(0.4)$ \\
\hline & $3-82$ & $0.04(0.02)$ & $0.005(0.001)$ & $0.001(0.001)$ & .. & $2.2(0.2)$ & . & $1.4(0.3)$ \\
\hline & $2 \cdot 83$ & $0.32(0.05)$ & $0.000(0.000)$ & $0.003(0.001)$ & . & $2.4(0.2)$ & $3.8(0.11)$ & $1.3(0.3)$ \\
\hline & $2-84$ & $0.00(0.10)$ & $0.002(0.001)$ & $-0.001(0.001)$ & .. & $2.1(0.2)$ & $3.5(0.30)$ & $7.8(0.8)$ \\
\hline & $3 \cdot 85$ & $0.01(0.06)$ & $0.000(0.001)$ & $0.001(0.001)$ & .. & $2.2(0.2)$ & $2.5(0.40)$ & $0.4(0.3)$ \\
\hline & $2 \cdot 86$ & $0.16(0.07)$ & $0.000(0.001)$ & $0.004(0.002)$ & $\cdots$ & $1.8(0.2)$ & $2.7(0.40)$ & $3.9(0.5)$ \\
\hline \multicolumn{9}{|l|}{$\underline{\text { Summary }}$} \\
\hline Number of Analyses & & 64 & 76 & 76 & 29 & 34 & 39 & 43 \\
\hline $\operatorname{Mean}(\bar{x})$ & & 0.43 & 0.001 & 0.007 & 0.34 & 2.4 & 3.2 & 2.6 \\
\hline Standard Deviation(s) & & 0.33 & 0.002 & 0.009 & 0.27 & 0.5 & 1.7 & 2.3 \\
\hline Minimum & & $0.00(0.10)$ & $0.005(0.002)$ & $.0 .002(0.010)$ & $0.03(0.03)$ & $1.3(0.2)$ & $.0 .2(0.06)$ & $-0.8(0.3)$ \\
\hline Maximum & & $1.4(0.08)$ & $0.010(0.006)$ & $0.052(0.006)$ & $1.0(1.2)$ & $3.9(0.2)$ & $8.5(0.30)$ & $8.8(0.9)$ \\
\hline
\end{tabular}


Appendix C

Sediment stations

\begin{tabular}{ll} 
Rio Chama & Year \\
\hline Chamita & \\
& $7 \cdot 74$ \\
& $5 \cdot 75$ \\
& $9 \cdot 75$ \\
& $3 \cdot 76$ \\
& $10 \cdot 76$ \\
& $3 \cdot 77$ \\
& $10 \cdot 77$ \\
& $3 \cdot 78$ \\
& $3 \cdot 79$ \\
& $2 \cdot 80$ \\
& $3 \cdot 81$ \\
& \\
& $2 \cdot 83$ \\
& $2 \cdot 84$ \\
& $3 \cdot 85$ \\
& $2 \cdot 86$ \\
& \\
Rio Grande & $7 \cdot 74$ \\
Embudo & $7 \cdot 75$ \\
& $10 \cdot 75$ \\
& $3 \cdot 76$ \\
& $10 \cdot 76$ \\
& $3 \cdot 77$ \\
& $10 \cdot 77$ \\
& $3 \cdot 78$ \\
& $3 \cdot 79$ \\
& $2 \cdot 80$ \\
& $3 \cdot 81$ \\
& $3 \cdot 82$ \\
& $2 \cdot 83$ \\
& $2 \cdot 84$ \\
& $3 \cdot 85$ \\
& $2 \cdot 86$
\end{tabular}

Radiochemical Analyses of Sediments Showing Fallout and/or Hatural Levels

\begin{tabular}{|c|c|c|c|c|c|}
\hline $\begin{array}{r}{ }^{137} \mathrm{Cs} \\
(\mathrm{pCi} / \mathrm{g})\end{array}$ & $\begin{array}{l}238 \mathrm{Pu} \\
(\mathrm{pCi} / \mathrm{g})\end{array}$ & $\begin{array}{c}239,240_{\mathrm{Pu}} \\
(\mathrm{pCi} / \mathrm{g}) \\
\end{array}$ & $\begin{array}{c}90_{5 r} \\
(\mathrm{pcig} /)\end{array}$ & $\begin{array}{l}\text { Iotal Uranium } \\
-\quad(1 \mathrm{~g} / \mathrm{g})\end{array}$ & $\begin{array}{l}\text { Gross Gamma } \\
\text { (Counts/min/g) }\end{array}$ \\
\hline $0.08(0.12)$ & $0.000(0.001)$ & $0.001(0.001)$ & $\cdots$ & .. & . \\
\hline$\cdots$ & $0.002(0.002)$ & $0.001(0.001)$ & .. & - & .. \\
\hline - & $-0.001(0.001)$ & $0.001(0.001)$ & $\cdots$ & $\cdots$ & $\cdots$ \\
\hline $0.19(0.02)$ & $0.000(0.001)$ & $0.002(0.001)$ & . & -. & $\cdots$ \\
\hline - & $0.000(0.001)$ & $0.000(0.001)$ & $0.01(0.07)$ & .. & $\cdots$ \\
\hline $0.08(0.03)$ & $0.001(0.001)$ & $.0 .001(0.002)$ & $-0.01(0.06)$ & $\cdots$ & $\cdots$ \\
\hline $0.06(0.03)$ & $0.000(0.002)$ & $0.001(0.002)$ & $0.00(0.010)$ & . & - \\
\hline $0.00(0.03)$ & $0.000(0.001)$ & $.0 .002(0.0 \cap 2)$ & - & . & $1.3(0.11)$ \\
\hline $0.05(0.06)$ & $-0.091(0.002)$ & $0.006(0.001)$ & $-0.15(0.09)$ & $1.1(0.1)$ & $0.1(0.08)$ \\
\hline $0.18(0.03)$ & $0.001(0.001)$ & $0.003(0.001)$ & $0.82(0.11)$ & $1.5(0.2)$ & $1.7(0.11)$ \\
\hline $0.15(0.12)$ & $0.000(0.000)$ & $0.002(0.003)$ & $.0 .10(0.09)$ & -- & $1.3(0.11)$ \\
\hline . & . & -. & - & - & . \\
\hline $0.10(0.03)$ & $0.000(0.001)$ & $0.011(0.002)$ & $\cdots$ & $1.9(0.1)$ & $2.2(0.11)$ \\
\hline $0.27(0.15)$ & $0.000(0.001)$ & $0.000(0.001)$ & $\cdots$ & $1.3(0.1)$ & $2.0(0.10)$ \\
\hline $0.22(0.07)$ & $0.001(0.001)$ & $0.003(0.001)$ & .. & $1.9(0.2)$ & $1.6(0.30)$ \\
\hline $0.23(0.09)$ & $.0 .001(0.001)$ & $0.001(0.001)$ & $\cdots$ & $2.3(0.2)$ & $1.9(0.30)$ \\
\hline $0.56(0.13)$ & $0.001(0.002)$ & $0.005(0.002)$ & - & .. & .. \\
\hline - & $.0 .001(0.001)$ & $0.002(0.002)$ & $\cdots$ & . & . \\
\hline - & $0.000(0.001)$ & $0.011(0.002 ;$ & . & -. & $\cdots$ \\
\hline $0.14(0.02)$ & $0.000(0.001)$ & $0.002(0.001)$ & - & . & $\cdots$ \\
\hline - & $0.001(0.002)$ & $0.001(0.002)$ & $0.02(0.03)$ & - & . \\
\hline $0.14(0.02)$ & $0.001(0.002)$ & $0.002(0.004)$ & $.0 .04(0.06)$ & .. & . \\
\hline $0.09(0.03)$ & $-0.001(0.001)$ & $0.001(0.001)$ & $0.77(0.14)$ & - & - \\
\hline $0.26(0.08)$ & $.0 .002(0.001)$ & $-0.006(0.002)$ & . & - & $3.0(0.11)$ \\
\hline $0.24(0.10)$ & $.0 .002(0.007)$ & $0.004(0.001)$ & $0.07(0.07)$ & $3.0(0.3)$ & $3.1(0.11)$ \\
\hline $0.34(0.09)$ & $-0.002(0.002)$ & $0.004(0.002)$ & $0.10(0.04)$ & $4.6(0.5)$ & $5.8(0.13)$ \\
\hline $0.14(0.05)$ & $0.000(0.000)$ & $0.002(0.001)$ & $0.06(0.06)$ & . & $3.4(0.11)$ \\
\hline $0.23(0.04)$ & $0.001(0.000)$ & $0.002(0.001)$ & .. & $1.9(0.2)$ & $\cdots$ \\
\hline $0.12(0.22)$ & $0.000(0.001)$ & $0.002(0.001)$ & - & $2.3(0.1)$ & $2.4(0.11)$ \\
\hline $0.27(0.15)$ & $0.001(0.002)$ & $0.002(0.002)$ & $\cdots$ & $2.7(0.2)$ & $4.6(0.30)$ \\
\hline $0.09(0.07)$ & $-0.001(0.001)$ & $0.004(0.002)$ & . & $2.9(0.7)$ & $2.8(0.40)$ \\
\hline $0.05(0.06)$ & $0.000(0.002)$ & $0.003(0.001)$ & . & $2.8(0.3)$ & $3.1(0.40)$ \\
\hline
\end{tabular}


Appendix $C$ (cont)

\begin{tabular}{|c|c|c|c|}
\hline $\begin{array}{l}\text { Sediment Stations } \\
\text { (at) }\end{array}$ & Year & $\begin{array}{r}137 \mathrm{Cs} \\
(\mathrm{p} C \mathrm{i} / \mathrm{g})\end{array}$ & $\begin{array}{l}238 \mathrm{Pu} \\
(\mathrm{PCi} i / g)\end{array}$ \\
\hline Rio Grande & $10 \cdot 74$ & $0.16(0.12)$ & $0.000(0.000)$ \\
\hline \multirow[t]{15}{*}{ Otowi } & $5 \cdot 75$ & - & $0.000(0.000)$ \\
\hline & $10 \cdot 75$ & .. & $-0.002(0.001)$ \\
\hline & $4 \cdot 76$ & $0.20(0.02)$ & $.0 .001(0.001)$ \\
\hline & $10 \cdot 76$ & . & $0.000(0.001)$ \\
\hline & $3 \cdot 77$ & $0.06(0.03)$ & $0.003(0.002)$ \\
\hline & $10 \cdot 77$ & $0.08(0.08)$ & $0.000(0.001)$ \\
\hline & $3 \cdot 78$ & $0.08(0.02)$ & $0.000(0.001)$ \\
\hline & $3 \cdot 79$ & $0.11(0.02)$ & $0.000(0.046)$ \\
\hline & $2 \cdot 80$ & $0.09(0.05)$ & $0.000(0.001)$ \\
\hline & $3 \cdot 81$ & $0.14(0.05)$ & $0.001(0.001)$ \\
\hline & $3 \cdot 82$ & $0.16(0.03)$ & $0.002(0.001)$ \\
\hline & $2-83$ & $0.20(0.05)$ & $0.003(0.001)$ \\
\hline & $2 \cdot 84$ & $0.18(0.15)$ & $-0.001(0.002)$ \\
\hline & $3-85$ & $0.12(0.08)$ & $-0.001(0.001)$ \\
\hline & $2 \cdot 86$ & $0.09(0.07)$ & $0.001(0.001)$ \\
\hline Rio Grande & $9 \cdot 78$ & $0.13(0.03)$ & $-0.005(0.008)$ \\
\hline \multirow[t]{8}{*}{ Sandia } & $11 \cdot 79$ & $0.17(0.03)$ & $0.000(0.000)$ \\
\hline & $10 \cdot 80$ & $0.40(0.05)$ & $0.000(0.001)$ \\
\hline & 10.81 & $0.21(0.03)$ & . \\
\hline & $9 \cdot 82$ & $0.19(0.03)$ & $0.001(0.000)$ \\
\hline & $9 \cdot 83$ & $0.15(0.05)$ & $0.001(0.003)$ \\
\hline & $9 \cdot 84$ & $0.09(0.06)$ & $-0.006(0.003)$ \\
\hline & 9.85 & $0.23(0.08)$ & $0.000(0.001)$ \\
\hline & $10 \cdot 86$ & $0.17(0.07)$ & $0.000(0.001)$ \\
\hline Rio Grande & 9.78 & $0.07(0.03)$ & $.0 .005(0.008)$ \\
\hline \multirow{4}{*}{ Pajarito } & $11 \cdot 79$ & $0.08(0.05)$ & $0.000(0.000)$ \\
\hline & $10 \cdot 80$ & $0.05(0.04)$ & $0.001(0.001)$ \\
\hline & $10-81$ & $0.16(0.04)$ & . \\
\hline & 9.82 & $0.08(0.06)$ & . \\
\hline
\end{tabular}

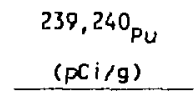

$0.032(0.008)$

$0.013(0.003)$

$0.013(0.011)$

$0.002(0.001)$

$0.003(0.002)$

$0.005(0.003)$

$0.003(0.002)$

$0.000(0.002)$

$0.023(0.046)$

$0.000(0.001)$

$0.000(0.001)$

$0.065(0.005)$

$0.002(0.001)$

$0.002(0.002)$

$0.003(0.002)$

$0.003(0.001)$

$-0.013(0.008)$

$0.004(0.000)$

$0.007(0.002)$

$0.011(0.001)$

$0.006(0.003)$

$0.002(0.004)$

$0.002(0.001)$

$0.002(0.001)$

$0.009(0.007)$

$0.001(0.000)$

$0003(0.001)$

\begin{tabular}{c}
${ }^{90} \mathrm{sr}$ \\
$-(p C i g /)$ \\
\hline \\
$\ldots$ \\
$\ldots$ \\
$\ldots$
\end{tabular}

$0.08(0.04)$

$0.51(0.10)$

$0.12(0.09)$

$0.42(0.08)$

$0.15(0.06)$

..

.

$\cdots$

-

$0.1,6(0.03)$

-

$\cdots$

.

-.

-.

-

$0.01(0.11)$

$0.24(0.03)$
Total Uranium ( $\mu g / 9)$

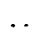

$\cdots$

.

.

-

$2.7(0.3)$

$2.6(0.2)$

..

$2.4(0.2)$

$3.0(0.2)$

$2.7(0.1)$

$2.6(0.2)$

$4.4(0.4)$

$2.2(0.2)$

$3.2(0.3)$

$2.4(0.2)$

$2.8(0.5)$

$2.9(0.2)$

$3.3(0.2)$

$2.9(0.3)$

-.

$1.9(0.2)$

$2.9(0.3)$
Gross Gamma

(Counts/min/g)

-.

.

$0.6(0.10)$

$3.4(0.12)$

$2.8(0.11)$

$3.7(0.11)$

$3.9(0.12)$

$4.3(0.30)$

$2.3(0.40)$

$5.2(0.40)$

$3.1(0.11)$

$3.7(0.12)$

-.

$3.4(0.11)$

$4.1(0.30)$

$4.2(0.50)$

$.5 .4(0.80)$

$1.5(0.11)$

$2.8(0.11)$ 


\begin{tabular}{|c|c|c|c|c|c|c|c|}
\hline $\begin{array}{l}\text { Sediment Stations } \\
\text { (at) }\end{array}$ & Year & $\begin{array}{r}137 \mathrm{cs} \\
(\mathrm{p} C \mathrm{i} / \mathrm{g}) \\
\end{array}$ & $\begin{array}{l}{ }^{23 B_{\mathrm{Pu}}} \\
(\mathrm{PCi} / \mathrm{g})\end{array}$ & $\begin{array}{c}239,240 \mathrm{Pu} \\
(\mathrm{pC} i / \mathrm{g})\end{array}$ & $\begin{array}{c}90 \mathrm{Sr} \\
(\mathrm{pC} i \mathrm{~g} /) \\
\end{array}$ & $\begin{array}{c}\text { Total Uranium } \\
(1 / g / g) \\
\end{array}$ & $\begin{array}{c}\text { Gross Gamma } \\
\text { (Counts/min/g) }\end{array}$ \\
\hline & $9 \cdot 83$ & $0.13(0.03)$ & $\cdot 0.002(0.002)$ & $0.001(0.003)$ & $\cdots$ & $2.8(0.5)$ & $3.5(0.11)$ \\
\hline & $9 \cdot 84$ & $0.09(0.08)$ & $-0.002(0.001)$ & $0.000(0.001)$ & $\cdots$ & $4.9(0.3)$ & $6.9(0.30)$ \\
\hline & $9 \cdot 85$ & $0.14(0.08)$ & $3.001(0.001)$ & $0.000(0.001)$ & $\cdots$ & $1.0(0.02)$ & $4.3(0.50)$ \\
\hline & $10 \cdot 86$ & $0.20(0.10)$ & $0.002(0.002)$ & $0.002(0.002)$ & $\cdots$ & $3.0(0.3)$ & $.4 .1(0.80)$ \\
\hline Rio Grande & 9.78 & $0.13(0.03)$ & $-0.006(0.013)$ & $0.003(0.010)$ & $\cdots$ & $\cdots$ & $\cdots$ \\
\hline \multirow[t]{8}{*}{ Ancho } & $11 \cdot 79$ & $0.32(0.05)$ & $0.000(0.000)$ & $0.008(0.000)$ & . & $2.9(0.2)$ & $4.3(0.12)$ \\
\hline & $10 \cdot 80$ & $0.30(0.04)$ & $-0.001(0.002)$ & $0.005(0.002)$ & $0.33(0.03)$ & $2.7(0.2)$ & $2.9(0.11)$ \\
\hline & $10 \cdot 81$ & $0.23(0.07)$ & - & -. & - & - & $\cdots$ \\
\hline & $9 \cdot 82$ & $0.16(0.04)$ & $0.000(0.000)$ & $0.015(0.001)$ & $\cdots$ & $\cdots$ & $\cdots$ \\
\hline & $9 \cdot 83$ & $0.04(0.06)$ & $.0 .003(0.004)$ & $\cdot 0.001(0.007)$ & $\cdots$ & $1.6(0.5)$ & $1.5(0.10)$ \\
\hline & $9 \cdot 84$ & $0.26(0.05)$ & $0.003(0.002)$ & $0.005(0.003)$ & $\cdots$ & $1.7(0.1)$ & $1.0(0.30)$ \\
\hline & $9 \cdot 85$ & $0.53(0.13)$ & $-0.002(0.002)$ & $0.018(0.003)$ & $\cdots$ & $1.4(0.1)$ & $4.8(0.60)$ \\
\hline & $10 \cdot 86$ & $0.13(0.07)$ & $-0.002(0.001)$ & $0.002(0.002)$ & $\cdots$ & $2.5(0.3)$ & $-4.8(0.80)$ \\
\hline Rio Grande & $9 \cdot 78$ & $0.15(0.03)$ & $0.012(0.010)$ & $-0.003(0.010)$ & - & $-\cdot$ & - \\
\hline \multirow[t]{8}{*}{ Frijoles } & $11 \cdot 79$ & $0.12(0.03)$ & $0.000(0.000)$ & $0.00 \bar{z}(0.000)$ & $-0.16(0.10)$ & $2.2(0.2)$ & $2.1(0.11)$ \\
\hline & $10 \cdot 80$ & $0.10(0.05)$ & $-0.001(0.001)$ & $0.004(0.002)$ & $0.25(0.03)$ & $2.6(0.3)$ & $2.0(0.11)$ \\
\hline & $10 \cdot 81$ & $0.20(0.07)$ & & $\cdots$ & $\cdots$ & - & - \\
\hline & $9 \cdot 82$ & $0.21(0.07)$ & - & $\cdots$ & $\cdots$ & - & $\cdots$ \\
\hline & $9 \cdot 83$ & $0.09(0.02)$ & $.0 .003(0.003)$ & $0.003(0.003)$ & $\cdots$ & $2.0(0.5)$ & $2.2(0.22)$ \\
\hline & $9 \cdot 84$ & $0.04(0.04)$ & $-0.003(0.002)$ & $0.000(0.001)$ & $\cdots$ & $1.9(0.1)$ & $1.5(0.30)$ \\
\hline & $\cdots$ & $\cdots$ & $\cdots$ & . & - & - & $\cdots$ \\
\hline & $10 \cdot 86$ & $0.28(0.10)$ & $0.001(0.001)$ & $0.009(0.002)$ & $\cdots$ & $3.6(0.4)$ & $0.5(0.60)$ \\
\hline Rio Grande & $7-74$ & $0.03(0.12)$ & $0.00 \cap(0.070)$ & $0.000(0.050)$ & $\cdots$ & $\cdots$ & $\cdots$ \\
\hline \multirow[t]{6}{*}{ Cochiti } & $5 \cdot 75$ & . & $0.0000(0.000)$ & $\cdot 0.001(0.001)$ & . & . & $\cdots$ \\
\hline & $10 \cdot 76$ & - & $0.003(0.002)$ & $0.002(0.002)$ & $0.90(0.30)$ & $\cdots$ & $\cdots$ \\
\hline & $3 \cdot 77$ & $0.05(0.02)$ & $.0 .020(0.030)$ & $0.030(0.020)$ & $0.03(0.01)$ & $\cdots$ & $\cdots$ \\
\hline & $10 \cdot 77$ & $0.04(0.03)$ & $0.000(0.002)$ & $0.001(0.001)$ & $.0 .05(0.09)$ & $\cdots$ & $\cdots$ \\
\hline & $3 \cdot 78$ & $0.03(0.05)$ & $.0 .001(0.002)$ & $0.001(0.002)$ & - & $\cdots$ & $\cdots$ \\
\hline & $11 \cdot 79$ & $0.29(0.05)$ & $0.001(0.002)$ & $0 . r 32(0.005)$ & $\cdots$ & $2.9(0.3)$ & $\cdots$ \\
\hline
\end{tabular}


Appendix $C$ (cont)

\begin{tabular}{cl}
$\begin{array}{c}\text { Sedi ent Stations } \\
\text { (at) }\end{array}$ & \\
\hline Rio Grande & \\
Bernalillo & $7 \cdot 74$ \\
& $5 \cdot 75$ \\
& $4 \cdot 76$ \\
& $10 \cdot 76$ \\
& $3 \cdot 77$ \\
& $10 \cdot 77$ \\
& $3 \cdot 78$ \\
& $3 \cdot 79$ \\
& $2 \cdot 80$ \\
& $3 \cdot 81$ \\
& $3 \cdot 82$ \\
& $2 \cdot 83$ \\
& $2 \cdot 84$ \\
& $\cdots$ \\
& $2 \cdot 86$ \\
& \\
& $7 \cdot 74$ \\
& $5 \cdot 75$ \\
& $9 \cdot 75$ \\
$4 \cdot 76$ \\
Jenez River & $10 \cdot 76$ \\
Jemez Pueblo & $3 \cdot 77$ \\
& $10 \cdot 77$ \\
& $3 \cdot 78$ \\
& $3 \cdot 79$ \\
& $2 \cdot 80$ \\
& $3 \cdot 81$ \\
& $3 \cdot 82$ \\
& $2 \cdot 8:$ \\
& $2 \cdot 84$ \\
& $3 \cdot 85$ \\
& $2 \cdot 86$
\end{tabular}

\begin{tabular}{|c|c|}
\hline $\begin{array}{c}137 \mathrm{Cs} \\
(p<i / g) \\
\end{array}$ & $\begin{array}{l}238 \mathrm{Pu} \\
(p \mathrm{C} i / g)\end{array}$ \\
\hline $0.23(0.12)$ & $0.000(0.000)$ \\
\hline$\cdots$ & $0.000(0.002)$ \\
\hline $0.23(0.02)$ & $-0.001(0.001)$ \\
\hline - & $0.000(0.001)$ \\
\hline $0.18(0.04)$ & $0.001(0.002)$ \\
\hline$\cdot 0.01(0.06)$ & $-0.004(0.001)$ \\
\hline $0.24(0.03)$ & $-0.001(0.002)$ \\
\hline $0.16(0.05)$ & $-0.000(0.001)$ \\
\hline $0.18(0.10)$ & $-0.002(0.002)$ \\
\hline $0.20(0.00)$ & $0.000(0.000)$ \\
\hline $0.39(0.03)$ & $0.001(0.001)$ \\
\hline $0.22(0.05)$ & $0.000(0.000)$ \\
\hline $0.25(0.15)$ & $-0.001(0.002)$ \\
\hline$\cdots$ & $\cdots$ \\
\hline $0.15(0.10)$ & $0.002(0.002)$ \\
\hline $0.34(0.12)$ & $0.000(0.001)$ \\
\hline . & $0.002(1.002)$ \\
\hline - & $-0.001(0.001)$ \\
\hline $0.13(0.02)$ & $0.001(0.001)$ \\
\hline$\ldots$ & $0.000(0.002)$ \\
\hline $0.32(0.04)$ & $0.000(0.001)$ \\
\hline $0.46(0.06)$ & $0.001(0.001)$ \\
\hline $0.26(0.07)$ & $0.000(0.002)$ \\
\hline $0.14(0.03)$ & $\cdot 0.001(0.002)$ \\
\hline $0.04(0.04)$ & $-0.002(0.001)$ \\
\hline $0.82(0.08)$ & $0.001(0.001)$ \\
\hline $0.29(0.08)$ & $-0.001(0.001)$ \\
\hline $0.16(0.06)$ & $0.001(0.000)$ \\
\hline $0.53(0.15)$ & $0.005(0.003)$ \\
\hline $0.18(0.08)$ & $0.000(0.001)$ \\
\hline $0.00(0.05)$ & $0.001(0.001)$ \\
\hline
\end{tabular}

\begin{tabular}{c}
239.240 Pu \\
(pCi $/ g)$ \\
\hline $0.004(0.001)$ \\
$0.004(0.002)$ \\
$0.005(0.001)$ \\
$0.003(0.001)$ \\
$0.013(0.007)$ \\
$0.001(0.001)$ \\
$-0.001(0.002)$ \\
$0.004(0.005)$ \\
$0.009(0.003)$ \\
$0.001(0.001)$ \\
$0.005(0.002)$ \\
$0.005(0.001)$ \\
$0.004(0.002)$ \\
.$\cdots$ \\
$0.013(0.003)$ \\
$0.002(0.001)$ \\
$0.001(0.001)$ \\
$0.001(0.001)$ \\
$0.002(0.001)$ \\
$0.008(0.002)$ \\
$0.006(0.002)$ \\
$0.007(0.002)$ \\
$0.002(0.002)$ \\
$0.001(0.002)$ \\
$0.000(0.003)$ \\
$0.015(0.003)$ \\
$0.002(0.001)$ \\
$0.003(0.001)$ \\
$0.010(0.003)$ \\
$0.002(0.001)$ \\
$0.002(0.001)$
\end{tabular}

\begin{tabular}{|c|c|c|}
\hline $\begin{array}{c}90 \\
\text { (pr } \mathrm{p} \text { ig/ })\end{array}$ & $\begin{array}{l}\text { Total Uranium } \\
(12 \mathrm{~g} / \mathrm{g})\end{array}$ & $\begin{array}{c}\text { Gross Gamma } \\
\text { (Counts/min/g) }\end{array}$ \\
\hline$\cdots$ & $\cdots$ & - \\
\hline . & $\cdots$ & - \\
\hline $0.80(0.10)$ & $\cdots$ & $\cdots$ \\
\hline $0.40(0.30)$ & - & $\cdots$ \\
\hline $0.05(0.02)$ & $\cdots$ & $\cdots$ \\
\hline $0.08(0.09)$ & . & . \\
\hline- & $5.8(0.8)$ & $3.2(0.11)$ \\
\hline$-\cdot$ & $3.2(0.3)$ & $3.3(0.12)$ \\
\hline $0.11(0.06)$ & - & $4.4(0.17)$ \\
\hline$\cdots$ & $3.1(0.3)$ & $0.2(0.10)$ \\
\hline . & $3.1(0.3)$ & -. \\
\hline - & $2.8(0.3)$ & $3.8(0.11)$ \\
\hline$\cdots$ & $2.7(0.1)$ & $4.2(0.30)$ \\
\hline - & - & - \\
\hline - & $2.9(0.3)$ & $3.0(0.40)$ \\
\hline . & $\cdots$ & $\cdots$ \\
\hline$\cdots$ & $\cdots$ & $\cdots$ \\
\hline - & $\cdots$ & $\cdots$ \\
\hline$\cdots$ & $\cdots$ & $\cdots$ \\
\hline . & $\cdots$ & $\cdots$ \\
\hline $0.16(0.08)$ & $\because$ & $\cdots$ \\
\hline $0.17(0.10)$ & $\cdots$ & . \\
\hline . & $\cdots$ & $8.6(0.15)$ \\
\hline - & $2.8(0.3)$ & $6.0(0.13)$ \\
\hline $1.1(0.06)$ & $2.0(0.2)$ & $5.2(0.12)$ \\
\hline $0.52(0.06)$ & - & $7.1(0.13)$ \\
\hline - & $3.0(0.3)$ & - \\
\hline - & $2.3(0.2)$ & $3.8(0.11)$ \\
\hline - & $4.1(0.2)$ & $8.4(0.30)$ \\
\hline$\cdots$ & $0.7(0.1)$ & $.0 .2(0.30)$ \\
\hline$\cdots$ & $1.9(0.2)$ & $4.1(0.50)$ \\
\hline
\end{tabular}


Sediment Stations
(at)

Surmary

Number of Analyses

Mcan $(\bar{x})$

Standard Deviation(s)

Minirnum

Maximum

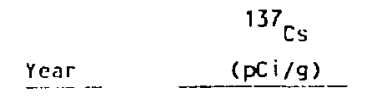

103

0.18

0.13

$.0 .01(0.06)$

$0.82(0.08)$

Appendix $C$ (cont)

Note: Analytical uncertainties within parentheses.

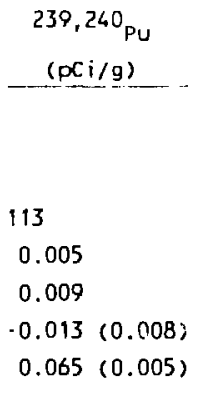

Total Uranium

$(\mu-g / g)$

36

0.23

0.32

$-0.16(0.10$ )

$1.1(0.06)$
2.6

0.9

$0.7(0.1)$

$5.8(0.8)$
Gross Gamma

(Counts/min/g)

62

2.9

2.5

$.0 .020(0.030)$

$0.012(0.010)$
$.4 .8(0.80)$

$8.6(0.15)$ 